\title{
Collagen content, but not the ratios of collagen type III/I mRNAs, differs among hypertensive, alcoholic, and idiopathic dilated cardiomyopathy
}

\author{
H.N. Soufen ${ }^{1}$, V.M.C. Salemi ${ }^{1}$, I.M.S. Aneas ${ }^{2}$, F.J.A. Ramires ${ }^{1}$, A.M.D. Benício ${ }^{3}$, \\ L.A. Benvenuti ${ }^{4}$, J.E. Krieger ${ }^{2}$ and C. Mady ${ }^{1}$ \\ ${ }^{1}$ Unidade de Cardiomiopatia, ${ }^{2}$ Laboratório de Genética e Cardiologia Molecular, ${ }^{3}$ Divisão de Cirurgia \\ Cardiotorácica, ${ }^{4}$ Laboratório de Patologia, Instituto do Coração, Hospital das Clínicas, Faculdade de \\ Medicina, Universidade de São Paulo, São Paulo, SP, Brasil
}

Correspondence to: V.M.C. Salemi, Avenida Jandira, 185, Apto. 41B, 04080-000 São Paulo, SP, Brasil Fax: +55-11-3556-9812. E-mail: verasalemi@uol.com.br

\begin{abstract}
Cardiac interstitial fibrosis may contribute to ventricular dysfunction and the prognosis of patients with dilated cardiomyopathy. The objective of the present study was to determine if total myocardial collagen content and collagen type III/I (III/I ratio) mRNAs differ in hypertensive, alcoholic, and idiopathic dilated cardiomyopathy subjects. Echocardiography and exercise cardiopulmonary testing were performed in patients with idiopathic $(N=22)$, hypertensive $(N=12)$, and alcoholic $(N=11)$ dilated cardiomyopathy. Morphometric analysis of collagen was performed in fragments obtained by endomyocardial biopsy with picrosirius red staining. The collagen III/I ratio was determined by reverse transcription polymerase chain reaction. Samples of controls $(\mathrm{N}=10)$ were obtained from autopsy. Echocardiographic variables and maximal oxygen uptake were not different among dilated cardiomyopathy groups. Collagen was higher in all dilated cardiomyopathy groups (idiopathic, hypertensive and alcoholic, $7.36 \pm 1.09 \%)$ versus controls $(1.12 \pm 0.18 \%), P<0.05$. Collagen was lower in idiopathic dilated cardiomyopathy $(4.97$ $\pm 0.83 \%)$ than hypertensive $(8.50 \pm 1.11 \%)$ and alcoholic $(10.77 \pm 2.09 \%)$ samples $(P<0.005$ for both $)$. The collagen III/I ratio in all samples from dilated cardiomyopathy patients was higher compared to that in controls $(0.29 \pm 0.04, P<0.05)$ but was the same in the samples from idiopathic $(0.77 \pm 0.07)$, hypertensive $(0.75 \pm 0.07)$, and alcoholic $(0.81 \pm 0.16)$ dilated cardiomyopathy groups. Because of the different physical properties of the types of collagen, the higher III/I ratio may contribute to progressive ventricular dilation and dysfunction in dilated cardiomyopathy patients.
\end{abstract}

Key words: Alcohol; Heart failure; Hypertension; Dilated cardiomyopathy; Collagen

H.N. Soufen was the recipient of a doctoral fellowship from FUNCOR-SBC. I.M.S. Aneas is the recipient of a doctoral fellowship from FAPESP (\#01/01295-7). J.E. Krieger is supported by grants from FAPESP (\#01/00009-0) and CNPq (\#471219/01-0).

Received January 21, 2008. Accepted November 25, 2008

\section{Introduction}

Left ventricular systolic dysfunction represents the final common pathway of several heart injury disorders (1). Despite recent major therapeutic advances, chronic heart failure still has a poor prognosis. Knowledge on the underlying cause of the cardiomyopathy has prognostic value (2-6). Mortality in patients with similar degrees of conges- tive heart failure and left ventricular dilatation due to alcoholic cardiomyopathy is significantly lower than that for patients with idiopathic cardiomyopathy (5). The cause of this difference is unknown.

In the normal adult heart, approximately $2-4 \%$ of the myocardium is collagen. Of the main collagen types, the major fibrillar collagens are types I and III, which are essential components of the cardiovascular extracellular 
matrix, maintaining myocardium structural and functional integrity (7-9). Collagen synthesis begins in the fibroblast. The disproportionate accumulation of fibrillar collagen in the interstitial space of the ventricle has been thought to be responsible for abnormal myocardial stiffness and for the impaired pumping capacity of the heart $(7,9,10)$. Because of their different physical properties, an altered collagen type III/I ratio (III/I ratio) may therefore have a major impact on the diastolic and systolic function of the heart and may influence the overall alterations occurring during the progression of the disease $(7,11)$. Whereas collagen type III represents about $11 \%$ of total collagen, and is characterized by thin fibers that present great elasticity (such as skin), collagen type I represents about $85 \%$ of total collagen, and is characterized by thick fibers that confer stiffness and resistance to stretch and deformation (such as tendon) (12).

A better understanding of the natural history of this remodeling process might permit better risk assessment and a more specific intervention earlier in this disease process, before it becomes irreversible. The increase in collagen content in hypertensive, alcoholic, and idiopathic dilated cardiomyopathy is well documented in studies of postmortem tissue $(12,13)$. However, because definitive data on collagen content and type in different forms of cardiomyopathy are limited, we determined the collagen content and the ratio of collagen subtype messenger RNAs (mRNAs) in endomyocardial biopsy samples from patients with dilated cardiomyopathy due to hypertension, alcoholism, and idiopathic cardiomyopathy and compared them with samples from the control group.

\section{Patients and Methods}

\section{Study cohort}

Forty-five consecutive patients [36 (80\%) males, $44 \pm$ 13 years] with dilated cardiomyopathy were selected for this study.

All patients were receiving optimized treatment with angiotensin-converting enzyme inhibitors and had left ventricular ejection fraction less than 0.50 with diffuse hypokinesis on echocardiography.

Patients were excluded from the study if one of the following criteria were met: coronary heart disease; cor pulmonale; valvular heart disease; pericardial disease; Chagas heart disease; diabetes mellitus; creatinine levels above $2 \mathrm{mg} / \mathrm{dL}$; coagulopathies; onset of cardiomyopathy during the last month of pregnancy or within 5 months of delivery; a history of treatment with cardiotoxic drugs; presence of active or borderline myocarditis in the endomyocardial biopsy fragments according to the Dallas crite- ria (14), and use of $\beta$-blockers, aldosterone antagonists, or spironolactone at any time before the study.

All procedures were carried out in accordance with institutional guidelines, and an Ethics Review Committee approved the protocol. All patients gave written informed consent before entering the study.

\section{Assignment of cause}

Patients were allocated into groups based on the cause of the cardiomyopathy. Idiopathic dilated cardiomyopathy was designated as the primary cause when no other cause of dysfunction was apparent after a full-noninvasive workup. Hypertensive cardiomyopathy was assigned as the primary cause when there was a history of poorly controlled hypertension of more than 5 years' duration. All study patients had a systolic blood pressure above $180 \mathrm{mmHg}$ or a diastolic blood pressure above $110 \mathrm{mmHg}$, or both of these. Alcoholic cardiomyopathy was assigned as the primary cause in the presence of heavy alcohol use, defined as the consumption of more than $100 \mathrm{~g} /$ day of ethanol for 6 months before the onset of heart failure.

\section{Endomyocardial biopsy}

An endomyocardial biopsy was performed on all patients with a Caves-Schultz bioptome to exclude myocarditis by way of the right internal jugular vein. In each case, three to ten fragments (mean of six fragments) were obtained from the interventricular septum of the right ventricle. One fragment was immediately placed in $10 \%$ formalin for light microscopy and picrosirius red staining. The fresh sample was immediately frozen.

\section{Duration of symptoms}

The duration of cardiac heart failure was defined as the time from the onset of subjective symptoms (dyspnea on exertion, paroxysmal nocturnal dyspnea, orthopnea and/ or edema) to the day of the endomyocardial biopsy.

\section{Echocardiography}

Left ventricular measurements were taken according to the recommendations of the American Society of Echocardiography (15). Measurements included left ventricular enddiastolic and end-systolic dimension obtained by M-mode echocardiography of the left ventricle in the short-axis view at the level of the papillary muscle. The cube of the enddiastolic dimension was used as an estimation of the enddiastolic volume and the cube of the end-systolic dimension as an estimation of end-systolic volume. Stroke volume was derived from the difference between end-diastolic and endsystolic volume. Ejection fraction was calculated as the ratio of stroke volume to end-diastolic volume. 


\section{Cardiopulmonary exercise testing}

Symptom-limited exercise testing together with a respiratory gas exchange analysis was performed using a bicycle ergometer (Medfit, Medical Fitness Equipment, Netherlands) at a constant pedaling speed of $60 \mathrm{rpm}$ with workload increments of $15 \mathrm{~W}$ every $3 \mathrm{~min}$ to measure the maximal oxygen uptake (16).

\section{Control group}

Hearts from 3 females and 7 males ( $48 \pm 13$ years) with no cardiac disease were sampled at necropsy. Right ventricular specimens were obtained from the interventricular septum within $10 \mathrm{~h}$ after death. All patients died from extra cardiac causes. The hearts were considered to be normal at necropsy. In addition, the samples obtained at necropsy were negative for RNA degradation measured by Northern blot analysis.

\section{Collagen quantification (collagen volume fraction)}

Histological sections of the endomyocardial biopsies and control samples (10 $\mu \mathrm{m}$ thick) were cut and stained with Sirius-red F3BA $(0.5 \%$ in saturated aqueous picric acid). A single investigator who was unaware of the nature of the experimental groups performed computer-assisted morphometry (Quantimet 520, Image Analysis System, Cambridge Instruments, UK). For each sample, all fields were analyzed $(6.7 \pm 2.8$ fields) with $10 \mathrm{X}$ objective lens under transmitted light. Collagen volume fraction (CVF) was calculated as the sum of all connective tissue areas divided by the sum of all connective tissue plus muscle areas in all fields. Collagen surrounding intramyocardial coronary arteries was excluded from the calculation. Another independent investigator randomly analyzed some of the samples. Intra- and interobserver variability were within $10 \%$. This morphometric approach for measurement of fibrillar collagen within the cardiac interstitium has been validated previously $(7,17)$. The total CVF is closely related to hydroxyproline content of the tissue $(18,19)$.

\section{Determination of the mRNAs of collagen types I and III}

The ratio of collagen type III/I mRNAs was determined by reverse transcription-polymerase chain reaction (RT-PCR).

Total RNA was isolated from frozen myocardial tissue by the gonidium acid thiocyanate/phenol/chloroform extraction method. mRNAs (300 ng) were reverse transcribed into cDNA with oligo (dT) and avian-myeloblastosis virus reverse transcriptase in a $20-\mu \mathrm{L}$ final volume. Amplification of $1 \mu \mathrm{L}$ of the reverse products was carried out with primers for the human $\alpha 1$ (III) collagen $(11,20)$ (upper primer 5'TGGTGTTGGAGCCGCTGCCA-3'; lower primer 5'CTCAGCACTAGAATCTGTCC-3'; product length 376 bp) and for human $\alpha 1$ (I) collagen (upper primer 5'-GGCGGCC AGGGCTCCGACCC-3'; lower primer 5'-ATTCCTGGTCT GGGGCACC-3'; product length $347 \mathrm{bp}$ ). Specificity of the amplified products was confirmed by sequencing. Primer sequences were selected to correspond to 2 different exons, separated by an intron, in order to permit identification of undesired genomic DNA amplification. The primers used for RT-PCR were located at the C-terminal end of the propeptides. In this region, the sequences are highly conserved. To make quantitative measurements of the mRNAs for collagen types I and III, cDNA for both collagen subtypes was co-amplified simultaneously in a single PCR. All PCRs were carried out in a $50-\mu \mathrm{L}$ volume. The mixture contained $25 \mathrm{pM}$ each of collagen primer, $1.25 \mathrm{mM}$ dNTP, $50 \mathrm{mM} \mathrm{KCl}, 2 \mathrm{mM} \mathrm{MgCl}$, $10 \mathrm{mM}$ Tris- $\mathrm{HCl}, \mathrm{pH} 9.0,0.1 \%$ Triton X-100, $2.5 \cup$ AmpliTaq polymerase. Each sample was incubated for 30 cycles, each cycle consisting of a denaturing step at $94^{\circ} \mathrm{C}$ for $1 \mathrm{~min}$, primer annealing at $58^{\circ} \mathrm{C}$ for $1 \mathrm{~min}$, and an extension at $72^{\circ} \mathrm{C}$ for $1 \mathrm{~min}$. After a final extension step at $72^{\circ} \mathrm{C}$ for $10 \mathrm{~min}$, the samples were stored at $4^{\circ} \mathrm{C}$. The proportion of synthesized RT-PCR products of collagen types III and I was constant for 25,30 , or 35 cycles of RT-PCR. Therefore, 30 cycles of the RT-PCR were used for further analysis. The PCR fragments were analyzed by polyacrylamide gel electrophoresis and visualized by Sybr gold staining. The band intensities of type III and type I collagens were quantified by densitometry scanning. The ratio of collagen types I and III was determined as the ratio of the density of corresponding mRNA bands.

\section{Statistical analysis}

Data are reported as means \pm SD. Data comparisons were made by analysis of variance. Post hoc pair-wise group comparisons were carried out with Duncan's multiple range test. The correlation between the characteristics of the myocardial interstitium (CVF and the relative amounts of the mRNAs of collagen subtypes III and I) and the left ventricular ejection fraction was determined with Pearson's correlation method. Significance level was assumed to be $P<0.05$. Statistical analysis was performed with SPSS v 9 (SPSS Inc., USA).

\section{Results}

\section{Patient characteristics}

The 45 patients had a mean \pm SD ejection fraction of $34.1 \pm 0.88 \%$ and were assigned to the idiopathic dilated cardiomyopathy $(\mathrm{N}=22)$, hypertensive $(\mathrm{N}=12)$, or alcoholic heart disease $(\mathrm{N}=11)$ group according to the criteria described in Methods.

There were no differences among the three dilated 
cardiomyopathy groups regarding gender, age, duration of heart failure, end-diastolic and end-systolic diameter of the left ventricle or left ventricular ejection fraction, and maximal oxygen uptake. Baseline characteristics in the dilated cardiomyopathy and control groups are shown in Table 1. Routine histological analysis of the endomyocardial biopsy showed myocyte hypertrophy and interstitial fibrosis but no evidence of active or borderline myocarditis.

\section{Collagen volume fraction}

As expected, myocardial collagen volume fraction was higher in samples from patients with dilated cardiomyopathy from all causes $(7.36 \pm 1.09 \%)$ versus controls $(1.12 \pm$ $0.18 \%, P<0.05)$. However, the CVF differed among these groups $(P=0.0001)$, being lowest in idiopathic $(4.97 \pm$ $0.83)$ compared with hypertensive $(8.50 \pm 1.11 \%, P<$ $0.05)$ and alcoholic $(10.77 \pm 2.09 \%)$ samples $(P<0.05)$. Figure 1 shows interstitial collagen of myocardium fragments of idiopathic, hypertensive, and alcoholic dilated cardiomyopathies, and control groups.

\section{Collagen type I and III mRNAs}

The ratio of the mRNAs in the dilated cardiomyopathy samples from all causes was higher than that in controls $(0.29 \pm 0.04, P<0.05)$. There was no statistical difference in this ratio among the different cardiomyopathies $(0.77 \pm$ 0.07 for idiopathic; $0.75 \pm 0.07$ for hypertensive; $0.81 \pm$ 0.16 for alcoholic; $P>0.05$ ). Figure 2 shows gel electrophoresis of PCR products of three experiments.

Because the ratio of collagen type III/I mRNAs was higher in dilated cardiomyopathy, but was not different among the different cardiomyopathies, we determined whether the ratio correlated with left ventricle ejection fraction of the three groups of patients. Figure 3 shows the positive correlation between left ventricular ejection fraction and collagen III/I ratio mRNA in all dilated cardiomyopathy groups $(r=0.44, P=0.005)$. Similar analysis with total collagen volume fraction did not correlate with left ventricular ejection fraction ( $r=0.17, P=0.30$; data not shown).

Table 1. Baseline characteristics of the idiopathic, hypertensive, and alcoholic dilated cardiomyopathy and control groups in the present study.

\begin{tabular}{lcccc}
\hline Parameter & Idiopathic $(\mathrm{N}=22)$ & Hypertensive $(\mathrm{N}=12)$ & Alcoholic $(\mathrm{N}=11)$ & Control $(\mathrm{N}=10)$ \\
\hline Age (years) & $40.54 \pm 15.08$ & $52.08 \pm 11.31$ & $42.45 \pm 8.18$ & $48.00 \pm 13.01$ \\
Duration of symptoms (months) & $32.59 \pm 34.35$ & $52.17 \pm 48.33$ & $51.36 \pm 29.89$ & - \\
VO $_{2}$ max $\left(\mathrm{mL} \cdot \mathrm{kg}^{-1} \cdot \mathrm{min}^{-1}\right)$ & $11.38 \pm 2.77$ & $12.64 \pm 3.42$ & $11.26 \pm 2.28$ & - \\
LVEDV $(\mathrm{mL})$ & $71.41 \pm 7.72$ & $69.58 \pm 6.27$ & $75.63 \pm 11.37$ & - \\
LVESV $(\mathrm{mL})$ & $62.67 \pm 7.48$ & $59.75 \pm 6.34$ & $66.30 \pm 10.85$ & - \\
LVEF $(\%)$ & $32.23 \pm 5.33$ & $36.17 \pm 5.44$ & $35.81 \pm 6.64$ & - \\
\hline
\end{tabular}

Data are reported as means $\pm \mathrm{SD}$. $\mathrm{VO}_{2}$ max $=$ maximal oxygen uptake; LVEDV = left ventricle end-diastolic volume; LVESV $=$ left ventricle end-systolic volume; LVEF = left ventricle ejection fraction. ${ }^{*}$ There were no statistically significant differences of any of the variables among the groups (Kruskal-Wallis test and Duncan multiple range test).

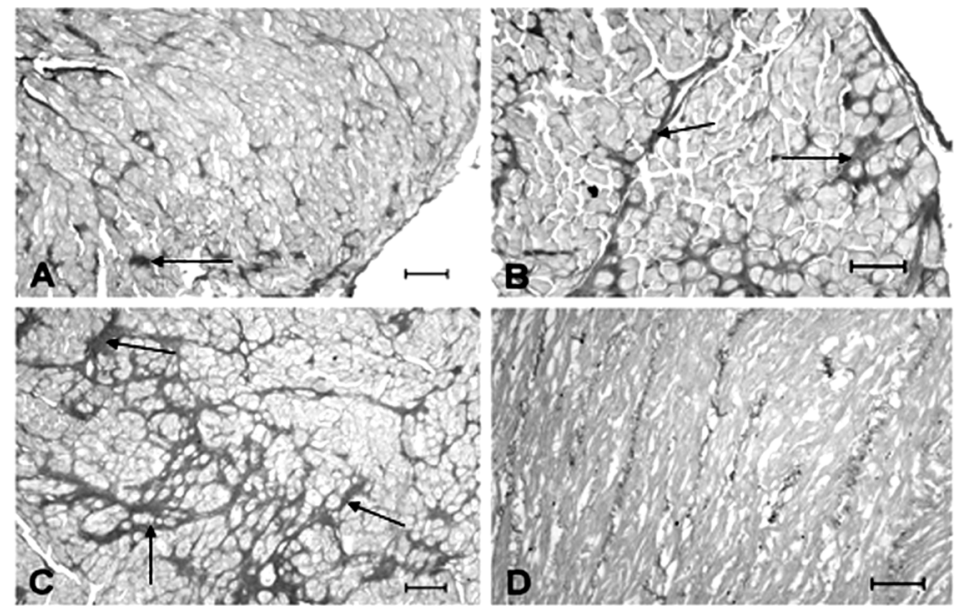

Figure 1. Representative fields of Sirius redstained sections of myocardium under $10 \mathrm{X}$ standard light magnification. $A$, Idiopathic; $B$, hypertensive; $C$, alcoholic; $D$, control. The interstitial collagen appears darker than myocytes in these black and white photographs (arrows). Scale bar $=100 \mu \mathrm{m}$ for all panels. 


\section{Discussion}

\section{Collagen quantification}

The myocardial collagen content in dilated cardiomyopathy samples was higher than that observed in control hearts, which agrees with previous data from postmortem tissues $(9,21)$. This occurred in spite of the use of high doses of angiotensin-converting enzyme inhibitors. Experimental studies show that these drugs are able to prevent and revert cardiac dysfunction and remodeling, even restoring myocardial structure to normal $(10,12,22-$ 24). The fact that all patients were receiving optimized treatment is consistent with the idea that the renin-angiotensin-aldosterone system is not the only determinant of myocardial fibrosis, stressing the importance of other modulating factors triggered by neurohumoral activation leading to myocyte necrosis and apoptosis. These results agree with the findings of Brilla et al. (25) showing that in 35 patients with hypertensive heart disease, the angiotensinconverting enzyme inhibitor treatment partially reduced the quantity of fibrosis, improved diastolic function, but CVF remained abnormal.

The collagen content of the samples of alcoholic and hypertensive cardiomyopathy patients was higher than that observed in patients with idiopathic dilated cardiomyopathy. Data from the literature show that, compared with patients with idiopathic dilated cardiomyopathy, patients with alcoholic and hypertensive cardiomyopathy have a better survival rate (5). One may speculate that the loss of collagen fibers from the matrix could lead to progressive dilation and impairment of cardiac pump performance, because the myocytes are not properly linked. Thus, under these conditions, less collagen content may be associated with a worse prognosis for cardiomyopathic hearts, pointing to a relevant role for metalloproteinases in this process $(26,27)$. Alterations in the interstitium are extremely complex, and the dynamic balance between the matrix synthesis and degradation may influence cardiac performance in a not so simple way. In addition, the higher amount of fibrosis observed in patients with alcoholic dilated cardiomyopathy may explain, at least in part, the higher prevalence of arrhythmia in these patients (28).

\section{Collagen type III/I expression ratio}

In this study, the myocardial ratio of collagen type III/I mRNAs of the dilated cardiomyopathy samples was higher than that observed in the normal myocardial tissue. This conversion in collagen types appears to be a characteristic feature of early collagen formation. In myocardial infarction, during early healing, the new collagen is mostly thin type III, and is susceptible to stretch by 15 weeks. Then, there is a replacement by type I with a greater resistance to distension $(29,30)$. In hypertensive myocardial fibrosis, there is an initial deposition of collagen type III, followed by type I $(12,13,31)$. Type III collagen is also increased in many experimental cardiac diseases $(7,14,32-36)$. In human cardiac samples, the results have been controversial. Weber et al. (21), studying hearts with idiopathic cardiomyopathy, observed a larger proportion of thin fibers, probably type III, which may contribute to dilation and loss of function. In contrast, the proportion between types III and I was reduced in samples obtained from explanted hearts with dilated cardiomyopathy (37), while cardiac samples

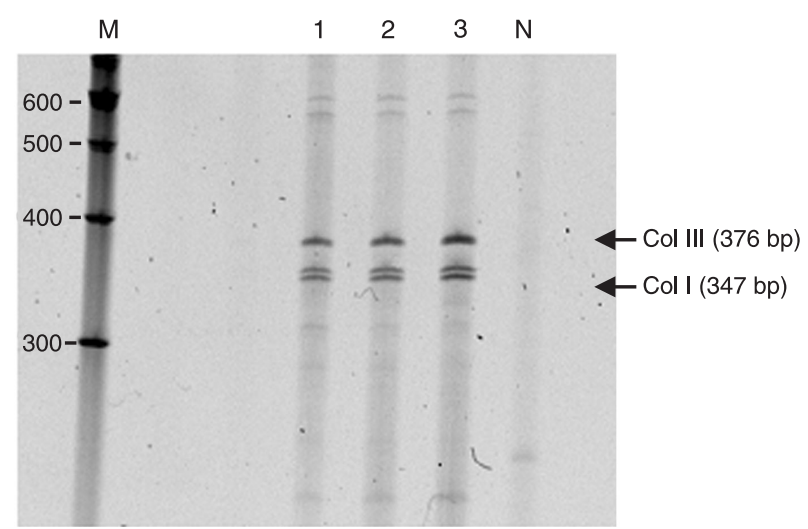

Figure 2. Gel electrophoresis of polymerase chain reaction products visualized by Sybr gold staining. M indicates DNA molecular weight markers. $\mathrm{N}$ indicates negative control. Col III, Col I = collagen types III and I. Data are representative of three experiments.

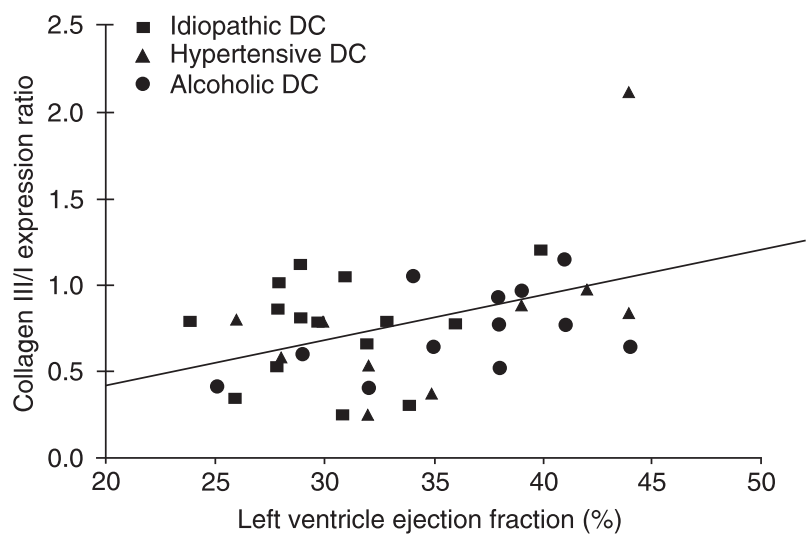

Figure 3. Linear regression between left ventricle ejection fraction and ratio of collagen type III/I mRNAs determined by RTPCR in all patients with dilated cardiomyopathy (DC; Pearson's correlation coefficient $=0.44, \mathrm{P}=0.005$ ). The different patient groups are indicated by squares for idiopathic, triangles for hypertensive, and circles for alcoholic disease. 
obtained at autopsy from hypertensive patients showed a disproportionate increase of collagen III (31).

In the present study, we found a positive correlation between the left ventricular ejection fraction and III/I ratio. Considering that all patients presented systolic dysfunction, we demonstrated that with more type I collagen, which is associated with strength and stiffness, the left ventricular ejection fraction is worse. On the other hand, a previous study of dilated cardiomyopathy with left ventricular ejection fraction lower than $50 \%$ presented increased I/III ratio, and related this finding to systolic and diastolic dysfunction (38). What seems to be contradictory, at first analysis, is that the normal myocardium had a lower proportion of collagen III/I mRNA compared with that in the dilated cardiomyopathy groups. Based on these results, it is possible that with dilated cardiomyopathy evolution, a reduc- tion in proportion between collagens III and I occurs, but this proportion is never equal to that observed in normal tissue. In other words, the scar matures, but can never complete this process, because new aggressive stimuli may trigger an endless new cycle.

Based on our results, the next step is the application of this information for new therapeutic interventions.

\section{Limitations of this study}

A limitation of the present study is that the findings obtained from the analysis of the right ventricle endomyocardial biopsy samples may not represent the structural alterations taking place in the whole organ. However, data from the literature support the idea that, in dilated cardiomyopathy, the myocardial damage is homogeneous, thus reducing the weight of such a limitation (39).

\section{References}

1. Hunt SA, Abraham WT, Chin MH, Feldman AM, Francis GS, Ganiats TG, et al. ACC/AHA 2005 Guideline Update for the Diagnosis and Management of Chronic Heart Failure in the Adult: a report of the American College of Cardiology/American Heart Association Task Force on Practice Guidelines (Writing Committee to Update the 2001 Guidelines for the Evaluation and Management of Heart Failure): developed in collaboration with the American College of Chest Physicians and the International Society for Heart and Lung Transplantation: endorsed by the Heart Rhythm Society. Circulation 2005; 112: e154-e235.

2. Adams KF Jr, Dunlap SH, Sueta CA, Clarke SW, Patterson $\mathrm{JH}$, Blauwet $\mathrm{MB}$, et al. Relation between gender, etiology and survival in patients with symptomatic heart failure. $J \mathrm{Am}$ Coll Cardiol 1996; 28: 1781-1788.

3. Fonarow GC. Epidemiology and risk stratification in acute heart failure. Am Heart J 2008; 155: 200-207.

4. Felker GM, Thompson RE, Hare JM, Hruban RH, Clemetson $\mathrm{DE}$, Howard DL, et al. Underlying causes and long-term survival in patients with initially unexplained cardiomyopathy. N Engl J Med 2000; 342: 1077-1084.

5. Prazak P, Pfisterer M, Osswald S, Buser P, Burkart F. Differences of disease progression in congestive heart failure due to alcoholic as compared to idiopathic dilated cardiomyopathy. Eur Heart J 1996; 17: 251-257.

6. Nagarakanti R, Whellan D, Rubin S, Mather PJ. Reversible cardiomyopathies. Cardiol Rev 2007; 15: 178-183.

7. Weber KT, Janicki JS, Shroff SG, Pick R, Chen RM, Bashey RI. Collagen remodeling of the pressure-overloaded, hypertrophied nonhuman primate myocardium. Circ Res 1988; 62: 757-765.

8. Medugorac I, Jacob R. Characterisation of left ventricular collagen in the rat. Cardiovasc Res 1983; 17: 15-21.

9. Brower GL, Gardner JD, Forman MF, Murray DB, Voloshenyuk T, Levick SP, et al. The relationship between myocardial extracellular matrix remodeling and ventricular function. Eur J Cardiothorac Surg 2006; 30: 604-610.

10. Jugdutt BI. Ventricular remodeling after infarction and the extracellular collagen matrix: when is enough enough? Circulation 2003; 108: 1395-1403.

11. de Souza RR. Aging of myocardial collagen. Biogerontology 2002; 3: 325-335.

12. Tayebjee $\mathrm{MH}$, MacFadyen RJ, Lip GY. Extracellular matrix biology: a new frontier in linking the pathology and therapy of hypertension? J Hypertens 2003; 21: 2211-2218.

13. Lopez SB, Ravassa AS, Arias GT, Gonzalez MA, Querejeta $\mathrm{R}$, Diez MJ. Altered fibrillar collagen metabolism in hypertensive heart failure. Current understanding and future prospects. Rev Esp Cardiol 2006; 59: 1047-1057.

14. Aretz HT, Billingham ME, Edwards WD, Factor SM, Fallon JT, Fenoglio JJ Jr, et al. Myocarditis. A histopathologic definition and classification. Am J Cardiovasc Pathol 1987; 1: 3-14.

15. Lang RM, Bierig M, Devereux RB, Flachskampf FA, Foster E, Pellikka PA, et al. Recommendations for chamber quantification: a report from the American Society of Echocardiography's Guidelines and Standards Committee and the Chamber Quantification Writing Group, developed in conjunction with the European Association of Echocardiography, a branch of the European Society of Cardiology. J Am Soc Echocardiogr 2005; 18: 1440-1463.

16. Albouaini K, Egred M, Alahmar A, Wright DJ. Cardiopulmonary exercise testing and its application. Heart 2007; 93: 1285-1292.

17. Weber KT, Pick R, Silver MA, Moe GW, Janicki JS, Zucker $\mathrm{IH}$, et al. Fibrillar collagen and remodeling of dilated canine left ventricle. Circulation 1990; 82: 1387-1401.

18. Weber KT, Clark WA, Janicki JS, Shroff SG. Physiologic versus pathologic hypertrophy and the pressure-overloaded myocardium. J Cardiovasc Pharmacol 1987; 10 (Suppl 6): 
S37-S50.

19. Nicoletti A, Heudes D, Hinglais N, Appay MD, Philippe M, Sassy-Prigent C, et al. Left ventricular fibrosis in renovascular hypertensive rats. Effect of losartan and spironolactone. Hypertension 1995; 26: 101-111.

20. Pauschinger $M$, Doerner A, Remppis $A$, Tannhauser $R$, Kuhl U, Schultheiss HP. Differential myocardial abundance of collagen type I and type III mRNA in dilated cardiomyopathy: effects of myocardial inflammation. Cardiovasc Res 1998; 37: 123-129.

21. Weber KT, Pick R, Janicki JS, Gadodia G, Lakier JB. Inadequate collagen tethers in dilated cardiopathy. Am Heart $J$ 1988; 116: 1641-1646.

22. Brilla CG, Janicki JS, Weber KT. Cardioreparative effects of lisinopril in rats with genetic hypertension and left ventricular hypertrophy. Circulation 1991; 83: 1771-1779.

23. Mulder P, Devaux B, Richard V, Henry JP, Wimart MC, Thibout $\mathrm{E}$, et al. Early versus delayed angiotensin-converting enzyme inhibition in experimental chronic heart failure. Effects on survival, hemodynamics, and cardiovascular remodeling. Circulation 1997; 95: 1314-1319.

24. Spinale FG, Holzgrefe HH, Mukherjee R, Hird RB, Walker JD, Arnim-Barker A, et al. Angiotensin-converting enzyme inhibition and the progression of congestive cardiomyopathy. Effects on left ventricular and myocyte structure and function. Circulation 1995; 92: 562-578.

25. Brilla CG, Funck RC, Rupp H. Lisinopril-mediated regression of myocardial fibrosis in patients with hypertensive heart disease. Circulation 2000; 102: 1388-1393.

26. Meier H, Bullinger J, Deten A, Marx G, Rabald S, Zimmer $\mathrm{HG}$, et al. Tissue inhibitor of matrix metalloproteinase-1 in norepinephrine-induced remodeling of the mouse heart. Cell Physiol Biochem 2007; 20: 825-836.

27. Van Linthout S, Seeland U, Riad A, Eckhardt O, Hohl M, Dhayat N, et al. Reduced MMP-2 activity contributes to cardiac fibrosis in experimental diabetic cardiomyopathy. Basic Res Cardiol 2008; 103: 319-327.

28. Vikhert AM, Tsiplenkova VG, Cherpachenko NM. Alcoholic cardiomyopathy and sudden cardiac death. J Am Coll Cardiol 1986; 8: 3A-11A.
29. Jugdutt BI. Left ventricular rupture threshold during the healing phase after myocardial infarction in the dog. Can $J$ Physiol Pharmacol 1987; 65: 307-316.

30. Yang Y, Ma Y, Han W, Li J, Xiang Y, Liu F, et al. Age-related differences in postinfarct left ventricular rupture and remodeling. Am J Physiol Heart Circ Physiol 2008; 294: H1815$\mathrm{H} 1822$.

31. Pardo Mindan FJ, Panizo A. Alterations in the extracellular matrix of the myocardium in essential hypertension. Eur Heart J 1993; 14 (Suppl J): 12-14.

32. Carver W, Nagpal ML, Nachtigal M, Borg TK, Terracio L. Collagen expression in mechanically stimulated cardiac fibroblasts. Circ Res 1991; 69: 116-122.

33. Masutomo K, Makino N, Sugano M, Miyamoto S, Hata T, Yanaga T. Extracellular matrix regulation in the development of Syrian cardiomyopathic Bio 14.6 and Bio 53.58 hamsters. J Mol Cell Cardiol 1999; 31: 1607-1615.

34. Eleftheriades EG, Durand JB, Ferguson AG, Engelmann GL, Jones SB, Samarel AM. Regulation of procollagen metabolism in the pressure-overloaded rat heart. J Clin Invest 1993; 91: 1113-1122.

35. Kruse JJ, Bart $\mathrm{Cl}$, Visser A, Wondergem J. Changes in transforming growth factor-beta (TGF-beta 1), procollagen types I and II mRNA in the rat heart after irradiation. Int $J$ Radiat Biol 1999; 75: 1429-1436.

36. Dawson R, Milne G, Williams RB. Changes in the collagen of rat heart in copper-deficiency-induced cardiac hypertrophy. Cardiovasc Res 1982; 16: 559-565.

37. Marijianowski MM, Teeling P, Mann J, Becker AE. Dilated cardiomyopathy is associated with an increase in the type l/ type III collagen ratio: a quantitative assessment. J Am Coll Cardiol 1995; 25: 1263-1272.

38. Pauschinger M, Knopf D, Petschauer S, Doerner A, Poller W, Schwimmbeck PL, et al. Dilated cardiomyopathy is associated with significant changes in collagen type $1 / I I$ ratio. Circulation 1999; 99: 2750-2756.

39. Beltrami CA, Finato N, Rocco M, Feruglio GA, Puricelli C, Cigola E, et al. The cellular basis of dilated cardiomyopathy in humans. J Mol Cell Cardiol 1995; 27: 291-305. 